\title{
LA TRANSFORMACIÓN DE LA UNIVERSIDAD CATÓLICA DE SANTIAGO DE GUAYAQUIL, CASO INÉDITO EN LAS UNIVERSIDADES CATÓLICAS DE LATINOAMÉRICA
}

\author{
THE TRANSFORMATION OF THE CATHOLIC UNIVERSITY OF SANTIAGO DE \\ GUAYAQUIL, UNPRECEDENTED EVENT IN LATIN AMERICAN CATHOLIC \\ UNIVERSITIES
}

\section{GUILLERMO CÉSAR TAFUR AVILÉS}

Universidad Católica de Santiago de Guayaquil. guillermo.tafur@cu.ucsg.edu.ec

RESUMEN

La transformación estructural de la Universidad Católica de Santiago de Guayaquil, ocurrida entre 1972 - 1974, fue impulsada por iniciativa del movimiento estudiantil que evolucionó de una Universidad Católica Confesional a una Universidad Católica de Inspiración Cristiana, hecho inédito en las más de 200 universidades católicas de Latinoamérica. Este proceso transformador se desarrolla bajo la influencia de la Reforma Universitaria de Córdoba, Argentina, 1918; Movimientos Estudiantiles y Sociales de 1968, La situación de las universidades ecuatorianas en las décadas del 60 y 70 , así como también los pronunciamientos de la Iglesia Católica de esa época. El trabajo además espera demostrar que no existe una forma mágica de Gobernanza-Cogobierno Universitario, ya que cada Universidad debe buscar de acuerdo con sus características y realidades, la forma más eficiente de gobierno; en el Caso de la Universidad Católica de Santiago de Guayaquil el Cogobierno ha funcionado y es parte esencial en la realización de su Misión y Visión Institucional.

PALABRAS CLAVE: gobernanza, cogobierno, confesional, inspiración.
ABSTRACT

The structural transformation of the Catholic University of Guayaquil, between 1972 - 1974, was driven by the initiative of the student movement as it was to go from being a confessional Catholic University to a Christian Catholic University of Inspiration, unprecedented in the most 200 Catholic universities in Latin America. This transformation process takes place under the influence of the university reform in Cordoba, Argentina, 1918; Student and social movements of 1968, the situation of Ecuadorian universities in the $60 \mathrm{~s}$ and $70 \mathrm{~s}$, as well as the pronouncements of the Catholic Church at that time. The work also hopes to demonstrate that there is no magic way of governance-university co-governance, and that each University should look according to their characteristics and realities in the most efficient form of government; in the case of the Catholic University of Santiago de Guayaquil it has worked and co-governance is an essential part in carrying out their institutional mission and vision.

KEYWORDS: governance, co-government, confessional, inspiration. 
INTRODUCCIÓN

Los hechos históricos e ideologías predominantes que le tocó vivir a la dirigencia estudiantil entre los años 60 y 70, sirvieron de antecedentes en su formación, por eso se hace alusión a la Reforma de Córdoba, donde se plantearon conceptos que incluso hoy están vigentes como la autonomía universitaria, libertad de cátedra, y cogobierno, entre otros.

Además se consideran los hechos históricos ocurridos en 1968, como la muerte de Martin Luther King y Robert Kennedy, movimientos sociales como la "Primavera de Praga" y el movimiento estudiantil de mayo de 1968 en Paris; así como los acontecimientos internos en la Iglesia Católica entre los que se destaca la promulgación del Documento de Buga, Colombia, "La misión de la universidad católica en América Latina”, promovido por el CELAM en 1967.

Adicionalmente se analiza el estado del movimiento estudiantil en el Ecuador en las décadas de los 60 y 70, donde además de la supresión de los exámenes de ingreso, se pedía el Cogobierno Paritario, la educación universitaria gratuita y la autonomía universitaria, entre otros.

Posteriormente se analiza la transformación de la Universidad Católica de Santiago de Guayaquil, la cual queda enunciada en el nuevo Estatuto de la Universidad, aprobado en la sesión Conjunta del Cuerpo de Cobierno y el Consejo Académico, realizada el 6 de diciembre de 1973, por la Comisión Episcopal Ecuatoriana, según comunicación dirigida por el Arzobispo de Guayaquil, monseñor César Antonio Mosquera Corral, el 27 de febrero de 1974, y puesto en vigencia por el Consejo Académico, en sesión del 28 de marzo de 1974.

Han pasado 46 años continuos de cogobierno y la Universidad Católica de Santiago de Guayaquil ha podido desarrollarse y cumplir un rol protagónico con la sociedad ecuatoriana, formando profesionales socialmente responsables, asegurando, al propio tiempo, de una manera institucional la impronta de una genuina inspiración cristiana y el mensaje de Cristo, tal como es transmitido por la Iglesia Católica, siendo los mismos principios señalados en la Ley Orgánica de Educación Superior del Ecuador (LOES, 2010), como autonomía responsable, cogobierno, igualdad de oportunidades, calidad, pertinencia, integralidad y autodeterminación, para la producción del pensamiento y conocimiento en el marco del diálogo de saberes, pensamiento universal y producción científica tecnológica global.

El objetivo es presentar el estado en que se encontraba la Universidad Latinoamericana y Ecuatoriana en la década de los 60 y 70, así como también el caso concreto de las universidades católicas del continente y el pronunciamiento de la Iglesia Católica acerca de la Educación Superior de la época, para poder analizar en ese marco, los hechos que conllevaron a la transformación de la Universidad Católica de Santiago de Guayaquil, de una Universidad Católica Confesional a una Universidad Católica de Inspiración, impulsada por el movimiento estudiantil.

Además, se espera demostrar que no existe una forma mágica de Gobernanza-Cogobierno universitario, ya que cada Universidad debe buscar, de acuerdo con sus características y realidades la forma más eficiente de gobierno; en el caso de la Universidad Católica de Santiago de Guayaquil se observa que después de 42 años de esa transformación ha funcionado y es parte esencial en la realización de su Misión y Visión Institucional.

\section{GOBIERNO, GOBERNABILIDAD Y GOBERNANZA UNIVERSITARIA}

El gobierno universitario es aquella estructura que ejerce la actividad de conducción, dirección y administración de la institución de educación superior. Dicho gobierno, en el caso del Ecuador, goza de la autonomía amparada en el texto constitucional de 2008 y en la Ley Orgánica de Educación Superior, LOES, de 2010.

La gobernanza, una de las formas de gobierno, caracterizada por una acción pública que conjuga la participación de todos, no de manera aislada sino concertada desde la definición de los problemas, la toma de decisiones y la ejecución de sus posibles soluciones, dando como valor agregado, no sólo la participación activa sino la responsabilidad de todos en la gestión de la acción.

Para que el gobierno universitario sea eficaz y legítimo, independientemente de la modalidad o forma de gobierno, es fundamental que se base en la gobernabilidad, considerada como la capacidad de gobernar de quienes ejercen la dirección de la institución de educación superior, cuyo fin es cumplir la misión encomendada, como libre ejercicio de la autonomía.

Se acoge las expresiones de José Joaquín Brunner de la Universidad Diego Portales de Chile, en su trabajo "Gobernanza universitaria: 
tipología, dinámicas y tendencias”, Brunner (2011) en el que manifiesta:

Por gobernanza se entiende aquí la manera en que las instituciones se hallan organizadas y son operadas internamente -desde el punto de vista de su gobierno y gestión- $y$ sus relaciones con entidades y actores externos con vistas a asegurar los objetivos de la educación superior. Se arranca con la hipótesis, común en la literatura especializada, de que las organizaciones universitarias exitosas son aquellas que logran modificar su gobernanza -esto es, el funcionamiento de su gobierno y sus formas de gestión- para adaptarla a las cambiantes demandas del entorno en que se desenvuelven y a las transformaciones estructurales de aquel. (p.137)

REFORMA UNIVERSITARIA DE 1918 CÓRDOBA

Se conoce por Reforma Universitaria de 1918, a la Reforma Universitaria de Córdoba, Reforma Universitaria de Argentina, o simplemente Reforma Universitaria (Universidad Nacional de Córdoba,1918), al movimiento estudiantil que se inició en la Universidad Nacional de Córdoba, Argentina en 1918, liderado por Deodoro Roca y otros dirigentes estudiantiles, y que se extendió luego a las demás universidades del país y de América Latina.

Principales postulados de la Reforma Universitaria de Córdoba fueron:

1. El cogobierno estudiantil.

2. La autonomía política, docente y administrativa de la universidad.

3. La elección de todos los mandatarios de la universidad por asambleas con representación de los profesores, de los estudiantes y de los egresados.

4. La selección del cuerpo docente a través de concursos públicos que aseguren la amplia libertad de acceso al magisterio.

5. La fijación de mandatos con plazo fijo (cinco años generalmente) para el ejercicio de la docencia, solo renovables mediante la apreciación de la eficiencia y competencia del profesor.

6. La gratuidad de la enseñanza superior.

7. La asunción por la universidad de responsabilidades políticas frente a la Nación y la defensa de la democracia.

8. La libertad docente.
9. La implantación de cátedras libres y la oportunidad de impartir cursos paralelos al del profesor catedrático, dando a los estudiantes la oportunidad de optar entre ambos.

10. La libre asistencia a las clases.

\section{MOVIMIENTOS ESTUDIANTILES Y SOCIALES DE 1968}

Las iniciativas emprendidas por los jóvenes del Barrio Latino de París corrió como pólvora por todo el planeta en un año revolucionario, 1968, que vio cómo los jóvenes luchaban por hacer realidad la Reforma Universitaria de 1918 y combatir a una sociedad corrupta en lugares como Berlín, México, Chicago o Praga.

Pareciera que en 1968 se concentró la gran revolución cultural, económica y política de la década de los años sesenta.

La Revolución de 1968, o simplemente el 68, es un término de gran éxito mediático, pero de difícil precisión historiográfica, incluso de debatida calificación como revolución. A veces se habla de ella como de un ciclo revolucionario (como el de la revolución de 1848 o la revolución de 1989), por la coincidencia temporal en el año 1968 del Mayo francés -que se suele considerar el epicentro del movimiento - con hechos y procesos similares.

Movimientos ligados a la revolución de 1968 pueden localizarse, entre otros países en Estados Unidos (protestas contra la Guerra de Vietnam, especialmente las que tuvieron lugar durante la Convención Nacional Demócrata de 1968, el Movimiento por los derechos civiles -de más amplio recorrido, pero que significativamente sufrió ese mismo año el asesinato de dos líderes significativos: Martin Luther King y Robert Kennedy-, el Festival de Woodstock -1969- y otros hechos y procesos relacionados), Checoslovaquia (Primavera de Praga, con su propuesta de socialismo de rostro humano; quizá el desencadenante o precipitante del movimiento parisino, mientras que su represión por los soviéticos significó una honda decepción en gran parte de la opinión progresista occidental), México (matanza de la plaza de Tlatelolco, coincidente con la celebración de los Juegos Olímpicos de México 1968).

A lo anteriormente expuesto ocho países en Sudamérica tienen dictadura militar, se fortalece la Revolución Cubana y líderes carismáticos como Fidel Castro, El Che Guevara y Salvador Allende entre otros, ejercen gran influencia en los movimientos estudiantiles de Latinoamérica. 


\section{LA EDUCACIÓN SUPERIOR EN EL ECUADOR, DÉCADAS DEL 60 Y 70 DEL SIGLO XX}

De 1960 hasta mediados de la década de 1970, Pareja, F. (CRESALC - UNESCO, 1986, p. 8-9), entra en crisis el sistema hacendario tradicional de la Sierra y se acelera el desarrollo capitalista del agro, se inicia la aplicación de una política coherente de industrialización, y se dinamiza el crecimiento urbano.

En general, el país entra en un acelerado proceso de modernización socioeconómica, que se reflejará claramente en la evolución de la educación superior. Adicionalmente, la Revolución Cubana primero, y la Alianza para el Progreso a continuación, ejercerán una influencia innegable en la vida universitaria.

La modernización del país y los dos fenómenos políticos anotados, influirán en la gestación de la reforma universitaria de los años sesenta, y en la extraordinaria proliferación de centros de educación superior y expansión de la matrícula.

La proliferación de universidades provinciales durante esos años, es consecuencia manifiesta de la creciente urbanización del país y el surgimiento de centros urbanos intermedios, en los que se hace presente una pequeña burguesía con legítimas aspiraciones de movilidad social a través del acceso a la educación superior. El establecimiento de centros de educación superior se convierte, asimismo, en un novedoso factor de prestigio para la ciudad y provincia, a la vez que factor de consolidación de formas más modernas de caudillismo local, como reflejo del proceso de modernización agropecuaria e industrial.

El impacto de la Revolución Cubana contribuyó decididamente a radicalizar el movimiento estudiantil, a politizar los centros universitarios. Durante los años sesenta se gesta y desarrolla un combativo movimiento de reforma universitaria liderado por la juventud estudiantil, al que se suman los afanes moderadamente reformistas de quienes, bajo el amparo de la Alianza para el Progreso, buscaban estructurar una universidad moderna al servicio del rápido desarrollo capitalista del país.

Otras reivindicaciones del movimiento de reforma, tales como el co-gobierno paritario estudiantil, la gratuidad de la enseñanza y la vinculación de la universidad con los sectores populares, aparecen en este contexto como resultado inevitable del grado de organización y combatividad adquirido por el movimiento estudiantil, la primera, y como recurso retórico de limitada concreción, la segunda.

Asimismo, las pequeñas universidades de provincia se caracterizan, salvo contadas excepciones, por un bajo nivel académico y una aguda escasez de recursos. Sólo las escuelas politécnicas de Quito y Guayaquil, así como las universidades administradas por la Iglesia Católica parecen haber eludido las manifestaciones más agudas de la crisis de los 60 y 70 en la Universidad Ecuatoriana.

\section{TIPOS DE UNIVERSIDADES CATÓLICAS UNIVERSIDADES CATÓLICAS ERIGIDAS O APROBADAS POR} LA SANTA SEDE

Son aquellas que son erigidas o aprobadas como tales por la Santa Sede, o por una conferencia episcopal, o por un arzobispo u obispo diocesano, de acuerdo con las normas aprobadas por la Santa Sede. La gran mayoría de estas instituciones, aunque son erigidas por aquella y reconocen como autoridad máxima al Papa, son puestas bajo la administración de la arquidiócesis o diócesis del lugar en que se encuentra su sede principal. De esta manera su autoridad directa e inmediata es el diocesano del lugar, quien ostenta la autoridad máxima de estos planteles ejerciendo el cargo de Gran Canciller. No obstante hay otras que dependen directamente de la Santa Sede (Precht, 2001).

Como signo de un mayor vínculo con la Iglesia, a una universidad católica le puede ser concedido, por la Congregación de Seminarios e Institutos de Estudios, el título honorífico de Pontificia. Esto sólo sucede en casos excepcionales y cuando la universidad tiene una larga tradición académica. Sólo hay 19 en el mundo, lo que es el caso de la Pontificia Universidad Católica de Chile o la Pontificia Universidad Católica del Ecuador. Su orgánico funcional no difiere de las simplemente llamadas universidades católicas.

Las que no portan el título honorífico de pontificia son simplemente llamadas universidades católicas, como por ejemplo la Universidad Católica de Cuenca que depende de la diócesis de Cuenca en Ecuador.

\section{UNIVERSIDADES CATÓLICAS ESTABLECIDAS POR OTROS ORGANISMOS ECLESIÁSTICOS}

También son católicas las universidades que dependen o son administradas por un instituto religioso u otra persona jurídica, que acepta en sus estatutos o documento equivalente, todo lo prescrito en relación a las universidades cató- 
licas. Este es el caso de muchas universidades mantenidas por órdenes religiosas, como por ejemplo los jesuitas, salesianos, etc.

Este tipo de planteles, al igual que las universidades católicas establecidas por la Santa Sede, también pueden recibir el título honorífico de pontificia como ocurre por ejemplo con la Pontificia Universidad Javeriana de Colombia que pertenece a la Compañía de Jesús.

\section{UNIVERSIDADES CATÓLICAS DE INSPIRACIÓN}

Son católicas también aquellas universidades que se definen como "católica de inspiración", en razón de un compromiso institucional hecho por las autoridades responsables de la institución. El establecimiento de una universidad de esta índole, debe recibir la aprobación del diocesano local y las autoridades deben informar acerca de sus actividades pastorales y su identidad católica al arzobispo u obispo de la diócesis en que está la sede principal de la institución, como sucede actualmente con la Universidad Católica de Santiago de Guayaquil.

EDUCACIÓN CATÓLICA EN AMÉRICA LATINA EN LAS DÉCADAS DEL 60 Y 70 DEL SIGLO XX

Se fortalece la "Teología de la Liberación" como corriente teológica que nace en el seno de la Iglesia católica en Latinoamérica tras el Concilio Vaticano II y la Conferencia de Medellín, teniendo grandes exponentes como monseñor Helder Cámara en Brasil y monseñor Leónidas Proaño en Ecuador.

Un hecho fundamental ocurre con el pronunciamiento de la Conferencia Episcopal de Latinoamérica, CELAM, en Buga, Colombia, en 1967 sobre las Universidades Católicas, aprobando las siguientes recomendaciones para mejorar las existentes (CELAM, 1967):

1. Contar con una facultad o centro de elaboración teológica de alto nivel, que realice el diálogo universitario entre teología y saber humano.

2. Procurar seriamente el mejoramiento académico de la Universidad, dando lugar a una dedicación de tiempo completo de la mayoría de sus profesores. Al mismo tiempo, debe garantizarse el reconocimiento efectivo "a los fieles, clérigos y laicos" de "la debida libertad de investigación, de pensamiento y de hacer conocer humilde y valerosamente, su manera de ver en el campo de su competencia".
3. Establecer una línea de prioridades respecto a las áreas culturales que han de ofrecerse en la Universidad. En este sentido, se han de tomar en cuenta las exigencias concretas locales. Especial atención merecen en este contexto, las ciencias sociales, matemáticas y de educación.

4. Revisar la estructura de poder, dando participación en el gobierno de institución y en la elección de sus autoridades, a los profesores y estudiantes, en todos los niveles. Esta revisión ha de llevar consigo la descentralización del poder. También debe reconocerse el legítimo derecho a sistemas organizativos y de representación por parte de los miembros integrantes de la comunidad universitaria.

5. La autonomía universitaria es un requisito indispensable para aquella libre investigación y expresión a la cual se ha hecho referencia.

Por esta razón, es necesario defender celosamente la autonomía de la comunidad universitaria frente a presiones estatales, a grupos políticos, empresariales y a fundaciones. Esta autonomía, sobre todo en el aspecto académico, ha de mantenerse también en las relaciones de la Universidad con la Jerarquía eclesiástica y los Superiores religiosos.

6. Promover la investigación y el estudio de aquellas áreas actualmente desatendidas y que están conectadas con el desarrollo social de América Latina, creando en cuanto sea posible, institutos de investigación de la realidad social, nacional o regional, institutos de estudios latinoamericanos, institutos para el estudio y promoción de la cultura popular latinoamericana.

7. Procurar eficazmente que el acceso a la Universidad esté abierto también a las clases menos favorecidas. Al respecto se señala la conveniencia de establecer un amplio sistema de préstamos.

8. Favorecer la interacción de la Universidad y la comunidad de la cual forma parte. De ahí se desprende la responsabilidad de la Universidad en las tareas de planeamiento del desarrollo de la misma comunidad. En forma específica, se señalan las tareas de prestación de servicios especializados, de acuerdo a las necesidades de la comunidad. 


\section{TRANSFORMACIÓN INÉDITA DE LA UNIVERSIDAD CATÓLICA DE SANTIAGO DE GUAYAQUIL}

Fundada el 17 de mayo de 1962, a petición de la Junta Pro Universidad Católica que presidía Mons. César Antonio Mosquera Corral, arzobispo de Guayaquil, el jurista Dr. Leonídas Ortega Moreira y el P. Joaquín Flor Vásconez S.J., quienes fueron sus autoridades fundadoras, como Gran Canciller, primer rector y consejero, respectivamente, siendo su tipología de Universidad Católica Confesional.

En sus diez primeros años la universidad fue creciendo hasta contar con 6 facultades. En 1972 los estudiantes plantean cambios como los de considerar la libertad de cátedra y el cogobierno universitario; sin embargo sus propuestas no son atendidas por lo que declaran una huelga indefinida el 13 de junio, la cual duraría cinco meses y tendría como hecho trascendental la toma del palacio Arzobispal el 13 de octubre siendo Bernardino Echeverría Ruiz, el arzobispo de la ciudad y Gran Canciller de la Universidad.

A raíz de este conflicto el arzobispo renuncia a su condición de autoridad de la Universidad y deja a la comunidad universitaria pronunciarse por el tipo de Universidad que considere.

Como resultado de un sinnúmero de reuniones y debates en el seno de la universidad, el 12 de septiembre de 1973 la comisión integrada por monseñor Francisco Larrea, representante alterno del Arzobispo; Rodrigo Malo González, representante alterno del Consejo Universitario; Dr. Galo García Feraud, representante principal del Cuerpo de Gobierno; Ab. Jacinto Loayza Mateus, representante alterno del Cuerpo de Gobierno y Guillermo Tafur Avilés, representante alterno de la Federación de Estudiantes, le entregan al Rector de la Universidad, Dr. José Baquerizo Maldonado, el proyecto del nuevo Estatuto de la Universidad para que los ponga a consideración y aprobación de los organismos pertinentes.

El nuevo Estatuto de la Universidad es aprobado en la sesión Conjunta del Cuerpo de Cobierno y el Consejo Académico realizada el día 6 de diciembre de 1973, por la Comisión Episcopal Ecuatoriana según comunicación dirigida por el Arzobispo de Guayaquil el 27 de febrero de 1974, y puesto en vigencia por el Consejo Académico, en sesión de 28 de marzo de 1974.

En el Estatuto queda claramente señalada la disposición de la Universidad de convertirse en una Universidad Católica de Inspiración como lo dice su Art. 1:
La Universidad Católica de Santiago de Guayaquil es un establecimiento de educación superior que, como tal, tiene como finalidades esenciales la preparación de profesionales socialmente responsables a base de investigación, conservación, promoción y difusión de la ciencia y de la cultura haciendo énfasis en sus valores autóctonos con miras a lograr el mejor desarrollo y superación del hombre ecuatoriano en un marco de convivencia democrática, justicia social, paz creadora, respeto y exaltación a los valores y derechos humanos, asegurando, al propio tiempo, de una manera institucional la impronta de una genuina inspiración cristiana y el mensaje de Cristo, tal como es trasmitido por la Iglesia Católica; la actuación comunitaria y trascendente, en un mundo universitario consciente de su función social y de frente a los problemas de la Sociedad Contemporánea; y el cumplimiento de su misión académica como Instituto de formación y cultura abierto a todas las corrientes del pensamiento universal.

Adicionalmente el Estatuto define claramente los principios de cogobierno y libertad de cátedra.

A partir de su aprobación, la Universidad institucionaliza el cogobierno universitario donde autoridades, profesores, estudiantes, y personal de administración y servicio, integran la comunidad universitaria. También se aprueban los principios de Autonomía, Libertad de Cátedra y Vinculación con la sociedad.

Finalmente, la comunidad universitaria decide cambiar la Tipología de Universidad $\mathrm{Ca}^{-}$ tólica, dejando de ser una Universidad Católica Confesional para pasar a ser una universidad Católica de Inspiración Cristiana, caso inédito en las universidades católicas de América Latina.

Con el logro de esta gran transformación ocurrida en la Universidad Católica de Santiago de Guayaquil, inédita en las Universidades Católicas de Latinoamérica y siendo uno de sus actores, planteo el reconocimiento de este hecho histórico que permitiría afirmar:

"La transformación de la Universidad Católica de Santiago de Guayaquil, 1973, es para las universidades católicas de América Latina, lo que la Reforma de la Universidad Nacional de Córdoba, 1918, es para las universidades estatales de Latinoamérica”. 
CONCLUSIONES

A través del movimiento estudiantil la Universidad Católica de Santiago de Guayaquil logró trascendentales cambios estructurales como el de pasar de ser una Universidad Católica Confesional a una Universidad Católica de Inspiración, hecho inédito en las más de 200 universidades católicas de Latinoamérica

En los 46 años continuos de cogobierno la Universidad Católica de Santiago de Guayaquil ha podido desarrollarse y cumplir un rol protagónico con la sociedad formando profesionales socialmente responsables, asegurando, al propio tiempo, de una manera institucional la impronta de una genuina inspiración Cristiana y el mensaje de Cristo, tal como es transmitido por la Iglesia Católica, siendo sus principios los mismos que se plantean en la Educación Superior del Ecuador señalados en el artículo 12 de la Ley Orgánica de Educación Superior (LOES; 2010) y que dice:

El Sistema de Educación Superior se regirá por los principios de autonomía responsable, cogobierno, igualdad de oportunidades, calidad, pertinencia, integralidad y autodeterminación para la producción del pensamiento y conocimiento en el marco del diálogo de saberes, pensamiento universal y producción científica tecnológica global.

Estos principios rigen de manera integral a las instituciones, actores, procesos, normas, recursos, y demás componentes del sistema, en los términos que establece esta Ley.

Por eso se puede decir, La Universidad Católica de Santiago de Guayaquil es una Universidad Católica de Inspiración y esto lo decidió la $\mathrm{Co}^{-}$ munidad Universitaria en 1974 al aprobar sus nuevos estatutos.

Por otra parte, se considera que no existe una forma mágica de Gobernanza-Cogobierno universitario; cada Universidad debe buscar, de acuerdo con sus características y realidades la forma más eficiente de gobierno; en el caso de la Universidad Católica de Santiago de Guayaquil el Co-Gobierno ha funcionado y es parte esencial en la realización de su Misión y Visión Institucional.

\section{REFERENCIAS BIBLIOGRÁFICAS}

Avilés, L. (2010). Cogobierno universitario y maquillaje numérico. Recuperado el 22 de octubre 2015 de http://www.80grados.net/cogobierno-universitarioy-maquillaje-numerico/\#sthash.rUWZMIiM.dpuf

Brunner, J. (2011) Gobernanza universitaria: tipología, dinámicas y tendencias, Universidad Diego Portales, Santiago, Chile. Revista de Educación, 355, Mayo-agosto 2011, pp.137-159, recuperado de: http://www.revistaeducacion.educacion.es/re355/ re355_06.pdf

Consejo Episcopal Latino Americano, CELAM, la misión de la universidad católica en américa... - CEPAU. Recuperado el 14 de octubre del 2015 de http:// www.cepau.org.ar/document/buga/buga1.pdf

Echeverría, P. Los movimientos estudiantiles de 1968 rompieron costumbres y métodos autoritarios, Recuperado el 14 deoctubre de 2015 de http://www. rebelion.org/noticia.php?id=92552

Fsc, H. F. H. C. P. (2007). La Universidad de La Salle: identidad cristiana y católica de su misión. Revista Universidad de La Salle, (44), 19-29. Recuperado de http://revistas.lasalle.edu.co/index.php/ls/article/ view/1680/1556
Pareja, F. Monografía La educación superior en el ecuador, Pág. 8,9, CRESALC - UNESCO, Caracas, Febrero 1986

Precht, j. (2001) Derecho Eclesiástico delo Estado de Chile. Ediciones Universidad Católica de Chile.

Ramírez. Tercera ola de transformación de la educación superior en Ecuador, Recuperado el 15 de octubre del 2015 http://reneramirez.ec/tercera-olade-transformacion-de-la-educacion-superior-enecuador/

Smalling, R. La Teología de la Liberación: Un Análisis, Recuperado el 12 de octubre del 2015 de La Teología de la Liberación: Un Análisis Roger L. Smalling, M.Å.

Universidad Nacional de Córdova, La Reforma Universitaria de 1918. Recuperado 30 de octubre del 2015 de http://www.unc.edu.ar/sobre-la-unc/historia/ reforma 